Pacific Journal of Mathematics

EXISTENCE OF BEST RATIONAL TCHEBYCHEF 


\section{EXISTENCE OF BEST RATIONAL TCHEBYCHEFF APPROXIMATIONS}

\section{BARRY BOEHM}

Some conditions are given which guarantee the existence of best Tchebycheff approximations to a given function $f$ by generalized rational functions of the form

$$
r(x)=\frac{a_{1} g_{1}(x)+\cdots+a_{n} g_{n}(x)}{b_{1} h_{1}(x)+\cdots+b_{m} h_{m}(x)}
$$

The principal theorem states that such a best Tchebycheff approximation exists whenever $f, g_{1}, \cdots, g_{n}, h_{1}, \cdots, h_{m}$ are bounded continuous functions, defined on an arbitrary topological space $X$, and the set $\left\{h_{1}, \cdots, h_{m}\right\}$ has the dense nonzezo property on $X:$ if $b_{1}, \cdots, b_{n}$ are real numbers not all zero, then the function $b_{1} h_{1}+\cdots+b_{m} h_{m}$ is different from zero on a set dense in $X$. An equivalent statement is that the set $\left\{h_{1}, \cdots, h_{m}\right\}$ is linearly independent on every open subset of $X$.

Further theorems assure the existence of best weighted Tchebycheff approximations and best constrained Tchebycheff approximations by generalized rational functions and by approximating functions of other similar forms.

Terminology. Let $X$ be an arbitrary topological space, and let $C[X]$ be the linear space of functions $f$ continuous on the space $X$, normed with the Tchebycheff norm

$$
\|f\|_{T^{\prime}}=\sup _{x \in X}|f(x)| .
$$

In this paper, we investigate the conditions necessary to guarantee the existence of a best approximation to functions $f \in C[X]$ by rational combinations of functions $g_{1}, \cdots, g_{n}, h_{1}, \cdots, h_{m} \in C[X]$. Such functions have the form

$$
r_{\gamma}=\frac{a_{1} g_{1}+\cdots+a_{n} g_{n}}{b_{1} h_{1}+\cdots+b_{m} h_{m}}
$$

where $\gamma=\left(a_{1}, \cdots, a_{n}, b_{1}, \cdots, b_{m}\right)$ is a vector in the closed set $\Gamma_{n+m}$ of all real $(n+m)$-tuples satisfying

Received March 12, 1964. The material in this paper was included in the author's Doctoral Dissertation submitted to the University of California, Los Angeles under the guidance of Professor E.W. Cheney. The research was supported in part by The RAND Corporation under U.S. Air Force Project RAND (Contract No. AF 49 (638)-700) and in part by the U.S. Air Force Office of Scientific Research (Contract No. AF-AFOSR-77-63). 


$$
\left|b_{1}\right|+\cdots+\left|b_{m}\right|=1 \text {. }
$$

One such condition is that $r_{\gamma}$ be well defined at points $x_{0}$ such that

$$
b_{1} h_{1}\left(x_{0}\right)+\cdots+b_{m} h_{m}\left(x_{0}\right)=0 ;
$$

thus, we shall restrict our attention to sets of functions $\left\{h_{1}, \cdots, h_{m}\right\}$ for which we can guarantee a unique definition of $r_{\gamma}\left(x_{0}\right)$.

A set of functions $\left\{h_{1}, \cdots, h_{m}\right\}$ is said to have the dense nonzero. property on $X$ if, for any $\gamma \in \Gamma_{n+m}$, the function

$$
b_{1} h_{1}+\cdots+b_{m} h_{m}
$$

is different from zero on a set $Y_{\gamma}$ dense in $X$. (An equivalent statement is that the set $\left\{h_{1}, \cdots, h_{m}\right\}$ is linearly independent on all open subsets of $X$.) If this is the case, the function $r_{\gamma}$ is well defined on the set $Y_{\gamma}$; to define $r_{\gamma}$ uniquely at points $x_{0} \in X-Y_{\gamma}$, we set

$$
r_{\gamma}\left(x_{0}\right)=\lim _{x \in r_{\gamma}, x \rightarrow x_{0}} \sup _{\gamma}(x) \text {. }
$$

We could define $r_{\gamma}\left(x_{0}\right)$ by a liminf operation just as well; all that is necessary is to define the function $r_{\gamma}$ uniquely, and in such a way that if the limit

$$
\lim _{x \in Y_{\gamma}, x \rightarrow x_{0}} r_{\gamma}(x)
$$

exists, it is equal to $r_{\gamma}\left(x_{0}\right)$. Thus, if $\left\{h_{1}, \cdots, h_{m}\right\}$ has the dense nonzero property on $X$, the generalized rational function $r_{\gamma}$ is uniquely defined on $X$ for all $\gamma \in \Gamma_{n+m}$.

For each set $\left\{g_{1}, \cdots, g_{n}, h_{1}, \cdots, h_{m}\right\}$ such that $\left\{h_{j}\right\}$ has the dense nonzero property on $X$, let $R$ denote the set of generalized rational functions

$$
R=\left\{r_{\gamma}: \gamma \in \Gamma_{n+m}\right\} .
$$

Then for each $f \in C[X]$ there exists a real number dist $(R, f)$ representing the distance from $f$ to the set $R$ :

$$
\operatorname{dist}(R, f)=\inf _{r_{\gamma} \in R}\left\|f-r_{\gamma}\right\| \text {. }
$$

If there exists a function $r_{\gamma *} \in R$ such that

$$
\left\|f-r_{\gamma *}\right\|=\operatorname{dist}(R, f),
$$

then $r_{\gamma *}$ is called a best rational approximation to $f$, and $\operatorname{dist}(R, f)$, is the error of the best rational approximation.

After a brief survey in 2 and 3 of previous existence results and nonexistence phenomena, we demonstrate in $\S 4$ that under the 
conditions prescribed above, there exists for every $f \in C[X]$ a best rational approximation $r_{\gamma *}$. Some extensions and specializations of this existence theorem, including its relation to the nonexistence phenomena of $\S 3$, will be given in $\S 5$. In $\S 6$, we present some existence theorems for two other approximating families similar in nature to the family of rational approximations.

2. Previous results. The special case $m=1, h_{1}(x)=1$ corresponds to approximation by generalized polynomials $a_{1} g_{1}+\cdots+a_{n} g_{n}$; it has been the subject of much fruitful study due to the feature of linearity in the coefficients $a_{i}$. An existence theorem was obtained in this case for Tchebycheff approximation of continuous functions $f$ by algebraic polynomials

$$
g_{i}(x)=x^{i-1}
$$

by Borel in 1905 [2]; his proof was extended by Achieser [1] to arbitrary elements $g_{i}$ in a normed linear space $S$.

Results are more sparse for the general rational problem $(m>1)$ in which the coefficients do not enter linearly. Walsh obtained in 1931 [6] an existence theorem for ratios of polynomials of the same degree defined on a perfect set $X$ in the complex plane.

THeOREM (Walsh). For any $f \in C[X], X$ a perfect set in the complex plane, there exists a best Tchebycheff approximation $r_{\gamma *}$ to $f$ among all rational functions of the form

$$
r_{\gamma}(x)=\frac{a_{0}+a_{1} x+\cdots+a_{n} x^{n}}{b_{0}+b_{1} x+\cdots+b_{n} x^{n}}
$$

for $\gamma \in \Gamma_{2 n+2}$.

Walsh also proved in [6] a similar existence theorem for $L^{p}$ norms. Achieser gives in [1] an incomplete proof of theorem above for ratios of polynomials of arbitrary degrees on an interval $[a, b]$ of the real line. Cheney and Loeb [3] have recently obtained a similar theorem for rational trigonometric approximation.

Furthermore, the Achieser and Cheney-Loeb theorems show that with no loss of generality the denominator of the best approximation may be assumed to be strictly positive on the interval of definition.

3. Nonexistence phenomena. Some of the possible pitfalls in the existence problem are illustrated by the following two examples of nonexistence phenomena. In the first example, we consider the problem of approximating $f(x)=x$ in the Tchebycheff sense by a rational function 
of the form

$$
r_{\gamma}(x)=\frac{a_{1} x^{2}}{b_{1}+b_{2} x}
$$

on the interval $[0,1]$, with the additional condition that the denominator be strictly positive on $[0,1]$. Here, however, by setting $a_{1}=b_{2}=1$ and letting $b_{1} \downarrow 0$, we see that $\operatorname{dist}(R, f)=0$, although no allowable $r_{\gamma} \in R$ achieves this minimum distance.

The second example shows that difficulties may arise when the dense nonzero property is violated. Consider the problem of approximating $f(x)=(x-1)(x-2) / 2$ in the Tchebycheff sense by a rational function of the form

$$
r_{\gamma}(x)=\frac{a_{1}}{b_{1}+b_{2} x}
$$

with the three points $0,1,2$ comprising $X$. Since $f(0)=1, f(1)=$ $f(2)=0$, we see that the deviation of the approximation $\varepsilon /(x+\varepsilon)$ from $f$ on $X$ is no greater than $\varepsilon /(1+\varepsilon)$, which can be made arbitrarily small by making $\varepsilon$ small. Thus $\operatorname{dist}(R, f)=0$, although again no choice of $r_{\gamma} \in R$ achieves this minimum.

4. An existence theorem. We shall find it convenient to state part of the theorem as a separate lemma.

LEMMA 1. If $f, h_{1}, \cdots, h_{m}$ are bounded functions on $X$, an arbitrary topological space, such that the set $\left\{h_{j}\right\}$ has the dense nonzero property on $X$, and if the set of functions $\left\{g_{1}, \cdots, g_{m}\right\}$ is linexrly independent on $X$, then any sequence $\left\{\gamma_{k}\right\}$ of vectors in $\Gamma_{n+m}$ such that

$$
\lim _{k \rightarrow \infty}\left\|r_{\gamma_{k}}-f\right\|=\inf _{\gamma \in \Gamma_{n+m}}\left\|r_{\gamma}-f\right\|=\operatorname{dist}(R, f),
$$

has a cluster point $\gamma_{0} \in \Gamma_{n+m}$.

Proof. (i). Define the functions $A=\sum a_{i} g_{i}, B=\sum b_{j} h_{j}$, with $\sum\left|b_{j}\right|=1$; define $A_{k}$ and $B_{k}$ similarly. The boundedness of the $h_{j}$ implies for any $B$ that

$$
\|B\| \leqq N=\max \left\|h_{j}\right\| ;
$$

the linear independence of the set $\left\{g_{i}\right\}$ implies the existence of a positive number $\delta$ such that

$$
\sum\left|a_{i}\right|=1 \text { implies }\|A\| \geqq \delta \text {. }
$$


It is clear that for sufficiently large $K, k \geqq K$ implies

$$
\operatorname{dist}(R, f)+1 \geqq\left\|r_{\gamma_{k}}\right\| \geqq \frac{\left\|A_{k}\right\|}{M}
$$

Hence, for $k \geqq K$

$$
\left\|A_{k}\right\| \leqq N[\operatorname{dist}(R, f)+1]
$$

and by the definition of the number $\delta$, for $k \geqq K$,

$$
\sum_{i=1}^{n}\left|a_{j_{k}}\right| \leqq M=\frac{N}{\delta}[\operatorname{dist}(R, f)+1]
$$

Thus, for $k \geqq K,\left\{\gamma_{k}\right\}$ is restricted to the compact set

$$
\left\{\gamma: \sum\left|a_{i}\right| \leqq M, \sum\left|b_{j}\right|=1\right\} \text {. }
$$

By the Bolzano-Weierstrass theorem, then, the sequence $\left\{\gamma_{k}\right\}$ has a cluster point $\gamma_{0} \in \Gamma_{n+m}$.

THEOREM 1. If $f, g_{1} \cdots, g_{n}, h_{1}, \cdots, h_{m}$ are bounded functions in $C[X], X$ an arbitrary topological space, and if the set $\left\{h_{j}\right\}$ has the dense nonzero property on $X$, then there exists a best rational Tchebycheff approximation $r_{\gamma *}$ to $f$ on $X$.

Proof. (i) Select a maximal linearily independent subset $\left\{g_{1}, \cdots, g_{p}\right\}$ among the functions $g_{i}$, and let $d=\operatorname{dist}(R, f)$. Then, any sequence $\left\{\gamma_{k}\right\}$ of vectors $\gamma_{k} \in \Gamma_{p+m}$ such that

$$
\left\|r_{\gamma_{k}}-f\right\| \leqq d+1 / k
$$

has by Lemma 1 a cluster point $\gamma_{0}=\left(a_{10}, \cdots, a_{p j}, b_{10}, \cdots, b_{m 0}\right) \in \Gamma_{p+m}$. We shall show that

$$
\left\|r_{\gamma_{0}}-f\right\|_{T}=d \text {. }
$$

Clearly, since $\gamma_{0} \in \Gamma_{p+m}$, we need only show

$$
\left\|r_{\gamma_{0}}-f\right\|_{T} \leqq d
$$

Since the set of functions $\left\{h_{j}\right\}$ has the dense nonzero property on $X$, the set $Y_{\gamma_{0}}$ of points $x$ at which the denominator $B_{0}(x)$ is different from zero, is dense in $X$. At points $x \in Y_{\gamma_{0}}$, we have for each $k$

$$
\begin{aligned}
\left|r_{\gamma_{0}}(x)-f(x)\right| & \leqq\left|r_{\gamma_{0}}(x)-r_{\gamma_{k}}(x)\right|+\left|r_{\gamma_{k}}(x)-f(x)\right| \\
& \leqq\left|r_{\gamma_{0}}(x)-r_{\gamma_{k}}(x)\right|+d+1 / k .
\end{aligned}
$$

As the functions $h_{j}$ are bounded on $X$, 


$$
B_{k} \underset{k \rightarrow \infty}{\longrightarrow} B_{0}
$$

uniformly on $X$. Since $B_{0}(x) \neq 0$ for $x \in Y_{\gamma_{0}}$, this implies

$$
\frac{A_{k}(x)}{B_{k}(x)} \underset{k \rightarrow \infty}{\longrightarrow} \frac{A_{0}(x)}{B_{0}(x)}
$$

for $x \in Y_{\gamma_{0}}$. Hence, for $x \in Y_{\gamma_{0}}$,

$$
\lim _{k \rightarrow \infty}\left|r_{\gamma_{0}}(x)-r_{\gamma_{k}}(x)\right|=0,
$$

and thus

$$
\left|r_{\gamma_{0}}(x)-f(x)\right| \leqq d .
$$

It remains only to obtain this inequality for points $x_{0} \in X-Y_{\gamma_{0}}$.

(ii). By the definition of the rational functions $r_{\gamma}$, we have for $x_{0} \in X-Y_{y_{0}}$ that

$$
r_{\gamma_{0}}\left(x_{0}\right)=\lim _{x \in Y_{\gamma_{0}}} \sup _{x \rightarrow x_{0}} r_{\gamma}(x) .
$$

Thus, there exists a sequence $\left\{x_{\nu}\right\}$ of points in $Y_{\gamma_{0}}$ such that

$$
\begin{aligned}
& \left|r_{\gamma_{0}}\left(x_{0}\right)-r_{\gamma_{0}}\left(x_{\nu}\right)\right| \leqq 1 / \nu \\
& \left|f\left(x_{0}\right)-f\left(x_{\nu}\right)\right| \leqq 1 / \nu
\end{aligned}
$$

(since also $f \in C[X]$ ). Hence,

$$
\begin{gathered}
\left|r_{\gamma_{0}}\left(x_{0}\right)-f\left(x_{0}\right)\right| \leqq\left|r_{\gamma_{0}}\left(x_{0}\right)-r_{\gamma_{0}}\left(x_{\nu}\right)\right|+\left|r_{\gamma_{0}}\left(x_{\nu}\right)-f\left(x_{\nu}\right)\right| \\
+\left|f\left(x_{\nu}\right)-f\left(x_{0}\right)\right| \leqq 1 / \nu+d+1 / \nu .
\end{gathered}
$$

Since the left hand side of this inequality is independent of $\nu$, it follows for $x_{0} \in X-Y_{\gamma_{0}}$ that

$$
\left|r_{\gamma_{0}}\left(x_{0}\right)-f\left(x_{0}\right)\right| \leqq d
$$

Therefore $\left\|r_{\gamma_{0}}-f\right\|_{T} \leqq d$, implying, since $\gamma_{0} \in \Gamma_{p+m}$, that $\left\|r_{\gamma_{0}}-f\right\|_{T}=d$, showing that indeed there exists a best approximation $r_{\gamma^{*}}=r_{\gamma_{0}}$ to $f$.

5. Extensions and specializations. Theorem 1 can be extended to the problem of weighted Tchebycheff approximation, in which the distance between $f$ and $r_{\gamma}$ is measured by the functional

$$
\left\|s\left(r_{\gamma}=f\right)\right\|_{T}
$$

for some prescribed weighting function $s \in C[X]$. This problem is equivalent to that of approximating the function $s f$ by rational combinations of the functions $s g_{1}$ and $h_{j}$; existence of a best approximation is thus guaranteed whenever the products $s f$ and $s g_{i}$ are bounded 
functions and the functions $h_{j}$ satisfy the hypotheses of Theorem 1 .

Also, the proof of Theorem 1 is valid if the coefficients $\gamma$ are restricted to a closed set $C_{n+m} \subset \Gamma_{n+m}$ containing at least one feasible vector $\gamma^{0}$ such that

$$
\left\|s\left(r_{\gamma^{0}}-f\right)\right\|_{T}<\infty .
$$

A slight but straightforward modification of step (ii) of Lemma 1 is needed if no vectors of the form $\left(0, \cdots, 0, b_{1}, \cdots, b_{m}\right)$ are in $C_{n+m}$.

Thus, the following theorem holds.

Theorem 2. If $f, s, g_{1}, \cdots, g_{n}, h_{1}, \cdots, h_{m} \in C[X]$ are such that the functions $s f, s g_{1}, \cdots, s g_{n}$ are bounded on $X$, an arbitrary topological space, and the set $\left\{h_{j}\right\}$ has the dense nonzero property on $X$, then for any closed set $C_{n+m} \subset \Gamma_{n+m}$ of coefficient vectors including a feasible vector $\gamma^{0}$, there exists a best weighted rational Tchebycheff approximation $r_{\gamma *}$ to $f$, such that

$$
\left\|s\left(r_{\gamma *}-f\right)\right\|_{T}=\inf _{\gamma \in O_{n+m}}\left\|s\left(r_{\gamma}-f\right)\right\|_{T} .
$$

If the closed set of coefficients $C_{n+m}$ of form

$$
C_{n+m}(\varepsilon)=\left\{\gamma \in \Gamma_{n+m}:\left|\sum b_{j} h_{j}(x)\right| \geqq \varepsilon>0, x \in X\right\}
$$

is nonempty, we can obtain existence theorems with much weaker hypotheses on the functions involved, since in this case the set $Y_{\gamma_{0}}$ comprises all of $X$, and step (ii) of Theorem 1 , the only step requiring the continuity of $f, s, g_{1}$, and $h_{j}$, is not required in the proof. Hence, the following theorem holds in an arbitrary normed linear space.

THEOREM 3. If the functions $f, s, g_{1}, \cdots, g_{n}, h_{1}, \cdots, h_{m}$ are such that $s f, s g_{1}, \cdots, s g_{n}, h_{1}, \cdots, h_{m}$ are bounded on $X$, an arbitrary set of points $x$, and if the set $C_{n+m}(\varepsilon) \subset \Gamma_{n+m}$ is nonempty, then there exists $a$ best weighted rational approximation $r_{\gamma *}$ to $f$ such that

$$
\left\|s\left(r_{\gamma *}-f\right)\right\|=\inf _{\gamma \in O_{n+m}(\varepsilon)}\left\|s\left(r_{\gamma}-f\right)\right\| .
$$

Let us now consider the nonexistence examples of $\S 3$ in the light of the above existence theorems. The first example can be handled by Theorem 1 by allowing the denominator $b_{1}+b_{2} x$ to have its zero at a point $x_{0} \in[0,1]$, and defining $a_{1} x_{0}^{2} /\left(b_{1}+b_{2} x_{0}\right)$ by a limsup operation, which reduces in this case to a limit opreration. Thus, the function $x^{2} / x$ is an acceptable rational function in Theorem 1 , and is indeed the best approximation $r_{\gamma *}$.

The second example cannot be handled by Theorem 1 since the dense nonzero property is violated. A weaker result can be given for 
both examples by Theorem 3, however, by considering only those rational functions such that $b_{1}+b_{2} x \geqq \varepsilon$; i.e., $\gamma \in C_{3}(\varepsilon)$. With this modification, a best approximation $r_{\gamma *}$ exists in the first example and is at least as good as $x^{2} /(\varepsilon+x)$; hence the error

$$
\operatorname{dist}(R, f) \leqq \varepsilon /(\varepsilon+1)
$$

can be made as small as desired by taking $\varepsilon$ small enough. In the second example, $r_{\gamma *}$ again exists and is at least as good as $\varepsilon /(\varepsilon+x)$; thus again

$$
\operatorname{dist}(R, f) \leqq \varepsilon /(\varepsilon+1) \text {. }
$$

In practical problems, placing such a "floor" under the denominator function and slightly above zero is often a reasonable thing to do, as the inequality constraint $B(x) \geqq \varepsilon$ is no harder to deal with than $B(x)>0$.

In most continuous rational Tchebycheff approximation problems, the existence of a best approximation is guaranteed by Theorems 1 and 2, as sets of functions with the dense nonzero property are fairly common. They include all linearly independent sets of functions analytic on a perfect set $X$, and all sets of piecewise analytic functions on $X$ which are linearly independent on each component of analyticity.

An independent result similar to Theorem 1 has been obtained recently by Newman and Shapiro [4]. Their existence theorem is stated for functions defined on a compact Hausdorff space $X$, and thus does not cover such problems as the approximation of functions continuous and bounded on the positive real axis by functions of the form

$$
r_{\gamma}(x)=\frac{\sum a_{i} e^{-\lambda_{i}} x}{\sum b_{i} e^{-\mu_{i}} x}
$$

for $\lambda_{i}, \mu_{j} \geqq 0$, a problem handled by Theorem 1 . Rice in [5] has also obtained independently a somewhat similar existence theorem for the interval $[0,1]$, under the assumption that the denominator possess only a finite set of zeros.

6. Existence theorems for other approximating families. The fact that best approximations exist among rational functions with coefficients in a closed set allows us, with the aid of the following lemma, to state some theorems assuring the existence of best approximations in other approximating families.

LEMmA 2. The set of all vectors $\left(c_{11}, \cdots, c_{1 m}, c_{21}, \cdots, c_{n m}\right)$ such that $c_{i j}=a_{i} b_{j}$ for real numbers $a_{i}, b_{j}$, is closed.

The proof of this lemma is straightforward, and is omitted here. 
The following theorem follows directly from Lemma 2 and Theorem 3 , with $m=1, n=p q$, and $g_{\nu}=u_{i} v_{j}$, since the set of numerator coefficients $c_{\nu}=a_{i} b_{j}$ is closed.

THEOREM 4. If the functions $f, s, u_{1}, \cdots, u_{p}, v_{1}, \cdots, v_{q}$ are such that the products $s f, s u_{1} v_{1}, \cdots, s u_{p} v_{q}$ are bounded on $X$, an arbitrary set of points $x$, then there exists a best approximation

$$
P^{*}=\left(a_{1}^{*} u_{1}+\cdots+a_{p}^{*} u_{p}\right)\left(b_{1}^{*} v_{1}+\cdots+b_{q}^{*} v_{q}\right)
$$

to the function $f$, such that

$$
\left\|s\left(P^{*}-f\right)\right\|=\inf _{a_{i}, b_{j}}\left\|s\left[\left(\sum a_{i} u_{i}\right)\left(\sum b_{j} v_{j}\right)-f\right]\right\| .
$$

In a similar fashion, a theorem can be established on the existence of best approximations by finite products of generalized polynomials of the form

$$
P=\left(\sum a_{i 1} g_{i 1}\right)\left(\sum a_{i 2} g_{i 2}\right) \cdots\left(\sum a_{i n} g_{i n}\right) .
$$

In particular, if the component polynomials are of the form $a x+b$, we have the following corollary.

Corollary 4a. Any function $f$ bounded on a compact domain $X$ on the real line has, among all polynomials $P_{n}$ of degree $n$ having only real roots, a best approximation $P_{n}^{*}$.

The next theorem follows from Lemma 2 and Theorem 2; a similar theorem can be based on Lemma 2 and Theorem 3 .

THEOREM 5. If the functions

$$
f, s, u_{1}, \cdots, u_{p}, v_{1}, \cdots, v_{q}, h_{1}, \cdots, h_{m} \in C[X]
$$

are such that the products of $s f, s u_{1} h_{1}, \cdots, s u_{p} h_{m}, s v_{1}, \cdots, s v_{q}$ are bounded on $X$, an arbitrary topological space, and the set $\left\{h_{j}\right\}$ has the dense nonzero property on $X$, then there exists a best weighted Tchebycheff approximation

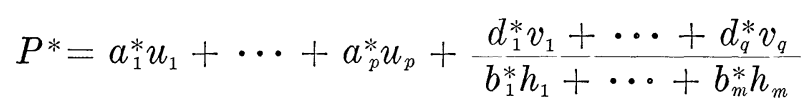

to the function $f$, such that

$$
\left\|s\left(P^{*}-f\right)\right\|_{T}=\inf _{a_{i} b_{j} a_{k}}\left\|s\left(\sum a_{i} u_{i}+\frac{\sum d_{k} v_{k}}{\sum b_{j} h_{j}}-f\right)\right\|_{T} .
$$




\section{REFERENCES}

1. N. I. Achieser, Theory of approximation, Frederick Ungar Publishing Company, New York, 1956.

2. E. Borel, Lecons sur les Fonctions des Variables Reelles, Paris, 1905.

3. E. W. Cheney, and H.L. Loeb, Generalized rational approximation, J. Soc. Indust. Appl. Math., Series B (Numerical Analysis) 1 (1964), 11-25.

4. D. J. Newman, and H. S. Shapiro, Approximation by generalized rational functions, Proceedings of Conference on Approximation, Birkhäuser Verlag, 1964 (to appear).

5. J. R. Rice, On the existence of best Tchebycheff approximations by general rational functions, Abstract 63T-331, Notices Amer. Math. Soc. 10 (1963), 576.

6. J. L. Walsh, The existence of rational functions of best approximation, Trans. Amer. Math. Soc. 33 (1931), 668-689.

The Rand Corporation 


\section{PACIFIC JOURNAL OF MATHEMATICS}

\section{EDITORS}

H. Samelson

Stanford University

Stanford, California

R. M. Blumenthal

University of Washington

Seattle, Washington 98105
J. Dugundu

University of Southern California Los Angeles, California 90007

*Richard Arens

University of California

Los Angeles, California 90024

\section{ASSOCIATE EDITORS}
E. F. BECKENBACH
B. H. NeumanN
F. WOLF
K. YOSIDA

\section{SUPPORTING INSTITUTIONS}

UNIVERSITY OF BRITISH COLUMBIA CALIFORNIA INSTITUTE OF TECHNOLOGY UNIVERSITY OF CALIFORNIA MONTANA STATE UNIVERSITY

UNIVERSITY OF NEVADA

NEW MEXICO STATE UNIVERSITY

OREGON STATE UNIVERSITY

UNIVERSITY OF OREGON

OSAKA UNIVERSITY

UNIVERSITY OF SOUTHERN CALIFORNIA
STANFORD UNIVERSITY

UNIVERSITY OF TOKYO

UNIVERSITY OF UTAH

WASHINGTON STATE UNIVERSITY

UNIVERSITY OF WASHINGTON

AMERICAN MATHEMATICAL SOCIETY CALIFORNIA RESEARCH CORPORATION SPACE TECHNOLOGY LABORATORIES NAVAL ORDNANCE TEST STATION 


\section{Pacific Journal of Mathematics}

\section{Vol. 15, No. $1 \quad$ September, 1965}

Donald Charles Benson, Unimodular solutions of infinite systems of linear

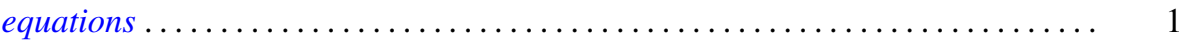

Richard Earl Block, Transitive groups of collineations on certain designs . . . . . . 13

Barry William Boehm, Existence of best rational Tchebycheff approximations .... . 19

Joseph Patrick Brannen, A note on Hausdorff's summation methods . . . . . . . . . . 29

Dennison Robert Brown, Topological semilattices on the two-cell ............ 35

Peter Southcott Bullen, Some inequalities for symmetric means . . . . . . . . . . 47

David Geoffrey Cantor, On arithmetic properties of coefficients of rational

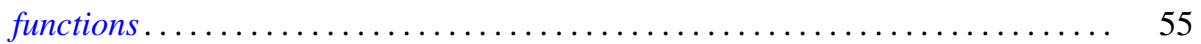

Luther Elic Claborn, Dedekind domains and rings of quotients . . . . . . . . . 59

Allan Clark, Homotopy commutativity and the Moore spectral sequence ........ 65

Allen Devinatz, The asymptotic nature of the solutions of certain linear systems of

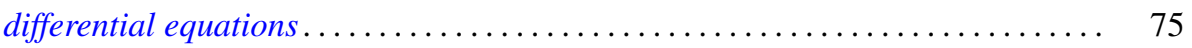

Robert E. Edwards, Approximation by convolutions ................... 85

Theodore William Gamelin, Decomposition theorems for Fredholm operators . . . . . 97

Edmond E. Granirer, On the invariant mean on topological semigroups and on

topological groups .................................. 107

Noel Justin Hicks, Closed vector fields . . . . . . . . . . . . . . . . . . . 141

Charles Ray Hobby and Ronald Pyke, Doubly stochastic operators obtained from

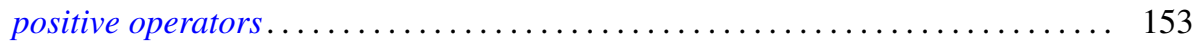

Robert Franklin Jolly, Concerning periodic subadditive functions . . . . . . . . . 159

Tosio Kato, Wave operators and unitary equivalence . . . . . . . . . . . . . . 171

Paul Katz and Ernst Gabor Straus, Infinite sums in algebraic structures . . . . . . . 181

Herbert Frederick Kreimer, Jr., On an extension of the Picard-Vessiot theory ...... 191

Radha Govinda Laha and Eugene Lukacs, On a linear form whose distribution is

identical with that of a monomial ......................... 207

Donald A. Ludwig, Singularities of superpositions of distributions . . . . . . . . . 215

Albert W. Marshall and Ingram Olkin, Norms and inequalities for condition

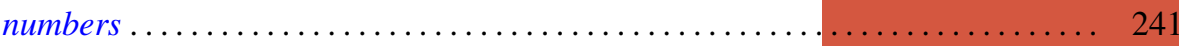

Horace Yomishi Mochizuki, Finitistic global dimension for rings . . . . . . . . . . 249

Robert Harvey Oehmke and Reuben Sandler, The collineation groups of division

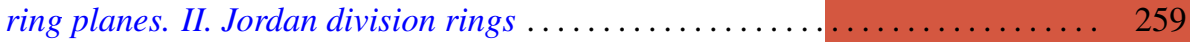

George H. Orland, On non-convex polyhedral surfaces in $E^{3} \ldots \ldots \ldots \ldots \ldots \ldots \ldots 267$

Theodore G. Ostrom, Collineation groups of semi-translation planes . . . . . . . . 273

Arthur Argyle Sagle, On anti-commutative algebras and general Lie triple

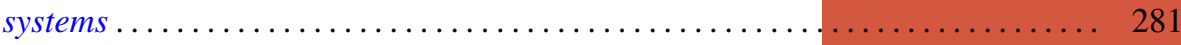

Laurent Siebenmann, A characterization of free projective planes . . . . . . . . . 293

Edward Silverman, Simple areas.................................. 299

James McLean Sloss, Chebyshev approximation to zero .................. 305

Robert S. Strichartz, Isometric isomorphisms of measure algebras . . . . . . . . . 315

Richard Joseph Turyn, Character sums and difference sets . . . . . . . . . . . . 319

L. E. Ward, Concerning Koch's theorem on the existence of arcs . . . . . . . . . . 347

Israel Zuckerman, A new measure of a partial differential field extension ......... 357 